Tropical Journal of Pharmaceutical Research December 2015; 14 (12): 2171-2178

ISSN: $1596-5996$ (print); 1596-9827 (electronic)

(c) Pharmacotherapy Group, Faculty of Pharmacy, University of Benin, Benin City, 300001 Nigeria.

All rights reserved.

Available online at http://www.tjpr.org

Original Research Article

http://dx.doi.org/10.4314/tjpr.v14i12.3

\title{
Structural and Dynamic Insight into Hirudin Epitopes-HLA- DRB1 0101 Complexes and their Modified Peptide Ligands: A Molecular Dynamic Simulation Study
}

\author{
Saeme Asgari ${ }^{1}$, Hasan Mirzahoseini ${ }^{2 \star}$, Morteza Karimipour ${ }^{3}$ and Azadeh \\ Ebrahim-Habibi ${ }^{4,5}$ \\ ${ }^{1}$ Department of Biology, Science and Research Branch, Islamic Azad University, ${ }^{2}$ Medical Biotechnology Department, \\ Biotechnology Research Center, Pasteur Institute of Iran, ${ }^{3}$ Molecular Medicine Department, Biotechnology Research Center, \\ Pasteur Institute of Iran, ${ }^{4}$ Biosensor Research Center, Endocrinology and Metabolism Molecular-Cellular Sciences Institute, \\ ${ }^{5}$ Endocrinology and Metabolism Research Center, Endocrinology and Metabolism Clinical Sciences Institute, Tehran University \\ of Medical Sciences, Tehran, Iran
}

${ }^{\star}$ For correspondence: Email: mirzahoseini@pasteur.ac.ir; Tel: +982166057220; Fax: +982166057220

Revised accepted: 26 October 2015

\begin{abstract}
Purpose: To develop a hirudin therapeutic protein that eliminates unwanted immune response. Methods: Molecular dynamic simulation was performed on immunodominant hirudin epitopes 1-15 and 13-27 and its analog, modified peptide ligands (MPLs), namely, [Lys $\left.{ }^{4}\right]$ Hir ${ }_{1-15}$ and [Gly $]$ Hir $1_{-15},\left[\mathrm{Gly}^{21}\right]$

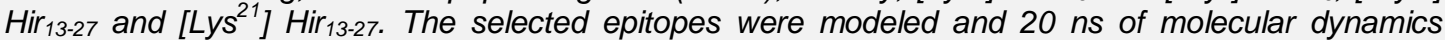
simulation was performed on peptide-HLA1 0101 and MPLs-HLA1 0101 complexes to gain a better understanding of molecular recognition mechanisms of MHC peptide binding. Characterization of the process was done by evaluation of root mean square deviation (RMSD) and total energy of binding. Result: All complexes of MPLs-HLA-DRB1 0101 showed thermodynamically unstable structure in comparison with native epitopes-HLA-DRB1 0101. The findings indicate that these analogs have different orientation in HLA grooves and are not available for suitable interaction with HLA-DRB1 0101. Conclusion: Altogether, the results show the potentials of predictive methods and molecular modeling in molecular mimicry of peptide-MHC interaction and provide insights into the binding characteristics of antigen presentation mechanism.
\end{abstract}

Keywords: Modified peptide ligand, Epitopes, MHC peptide binding, Hirudin, Modified peptide ligands, Molecular dynamic simulation, Binding free energy

Tropical Journal of Pharmaceutical Research is indexed by Science Citation Index (SciSearch), Scopus, International Pharmaceutical Abstract, Chemical Abstracts, Embase, Index Copernicus, EBSCO, African Index Medicus, JournalSeek, Journal Citation Reports/Science Edition, Directory of Open Access Journals (DOAJ), African Journal Online, Bioline International, Open-J-Gate and Pharmacy Abstracts

\section{INTRODUCTION}

Hirudin is a polypeptide consisting of $65-66$ amino acids. It is produced in the salivary glands of the leech Hirudo medicinalis and is the most potent thrombin inhibitor known which is naturally occurring [1-3]. R-hirudin is the currently accepted treatment for patients with heparininduced thrombocytopenia, but a significant problem in the clinical use of hirudin is its immunogenicity [4]. Hirudin has been shown to induce T-cell-dependent IgG antibody responses when used as a therapeutic protein [5].

Molecular dynamics (MD) simulation is a powerful technique, which provides a high resolution dynamic picture and has widespread use in modelling different biological molecular systems [6-8]. Previous MD simulations have been performed to investigate changes in 
conformational dynamics of thrombin binding cleft, when complexed with a native hirudin, and its mutants with single amino-acid mutation (submitted article). In the present study, four epitopes derived from mutants of hirudin variant III (HV3), have been studied with regard to their respective immunogenicity potential. We used immunoinformatic tools to antigen identification and characterization for the development of the hirudin, and obtained a successful prediction of the peptide-MHC binding. We have performed MD simulation runs of $20 \mathrm{~ns}$ to investigate the structural and dynamical differences of HLADRB1 0101 binding cleft, when complexed with a native peptide as well as the effect of single amino-acid mutation of the peptides.

This study focused on HLA-DRB1 0101 peptide binding site region to characterize and emphasize the similarities and differences in binding properties by (a) calculating root mean square deviation (RMSD) and (b) binding free energy. HLA-DRB1 0101 was selected because this haplotype has been observed to have increased frequency in Iran $[9,10]$.

\section{EXPERIMENTAL}

\section{In silico analysis of protein sequences epitope selection}

In a previous study, hirudin sequence was analyzed using two epitope prediction programs: IEDB (http://www.iedb.org/), and ProPred (http://www.imtech.res.in/raghava/propred/). In summary, the affinities of hirudin sequence were evaluated for HLA-DRB1 0101.The ProPred results include predicted MHC Class-II binding regions (9-mers core regions) in hirudin sequence and the results of the IEDB include binding affinity scores for fifteen-amino acid epitopes. IEDB program uses a maximum affinity score of fifty, and any affinity values below this score are considered significant. The epitopes with the highest affinities for HLA-DRB1 101 were selected. Fragments of fifteen amino acids, identified by the best alignment between the sequence of the entire protein and the epitopes previously selected were chosen $[6,11,12]$.

In silico mutations were performed for each position of the 9-mer binding core of the peptide, 19 mutated structures were generated where each structure contained a mutation of the core residue to one of the other 19 amino acids. Thus, for each epitope $171(19 \times 9)$ mutated structures were generated that covered all possible amino acids at each of the core position of the peptide [8].

\section{Molecular modeling methods}

The construction of the three-dimensional structures of the two selected epitopes (epitopes 1-15 and 13-27) and modified epitopes (MPLs,

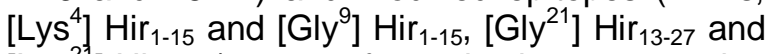
$\left[\mathrm{Lys}^{21}\right] \mathrm{Hir}_{13-27}$ ) was performed using comparative modeling, which is a technique that is used to construct three-dimensional protein structures based on structurally similar proteins that have an evolutionary relationship. The Modeller9v13 [13] program was used to construct the threedimensional structures of the epitopes using a structural alignment between the target sequence and the region of similarity of the protein template (PDB code: 4HTC) [14]. The best model was selected based on its energy, and it was subjected to further 20 ns molecular dynamics using Gromacs 4.6.5 Software package [15]. Epitopes and the mutated peptides structures were minimized before they were subjected to binding free energy calculation.

\section{Molecular dynamics simulation}

The initial structure of epitopes and its mutants were obtained from homology modeling. To obtain the starting structure of the epitopes/MHC class II (DRB1*0101, PDB code: 1PYW) [16] complex for molecular dynamics (MD) simulation, the docking protocol consists of rigid-body docking, semi-flexible refinement stage and final refinement inexplicit solvent was performed [17]. All of the simulations and the analysis of the trajectories were performed with Gromacs 4.6.5 software package using the Gromos 96 force field software package. Each starting structure was solvated with explicit water molecules in a periodic cubic box. GENION procedure of Gromacs was used to obtain a neutral system by replacing the water molecules with $\mathrm{Na}^{+}$ions. For native epitopes, [Gly $\left.{ }^{9}\right] \mathrm{Hir}_{1-15}$ and $\left[\mathrm{Gly}^{21}{ }^{2} \mathrm{Hir}_{13-27}\right.$, $3 \mathrm{Na}^{+}$ions were added, while $2 \mathrm{Na}^{+}$ions were added for $\left[\mathrm{Lys}^{4}\right] \mathrm{Hir}_{1-15}$ and $\left[\mathrm{Lys}^{21}\right] \mathrm{Hir}_{13-27}$.

The native epitopes/DRB $1^{*} 0101$ and mutated epitopes/DRB1*0101 systems were first energy relaxed with 250000 steps of steepest-descent energy minimization without position restraints. The equilibration run was followed by a 20 ns MD run without position restraints. The equilibration of the trajectory was checked by monitoring RMSD with respect to the initial structure and the free binding energy. The electrostatic term was described with the particle mesh Ewald algorithm. LINCS algorithm was used to constrain all bond lengths. All simulations were performed at $300 \mathrm{~K}$. The density of the system was adjusted during the first equilibration runs at NPT condition by weak coupling to a bath of 
constant pressure $\left(P_{0}=1\right.$ bar, coupling time $T_{P}=$ $1 \mathrm{ps})$. The time step of the simulation was $2 \mathrm{fs}$. Electrostatic interactions were calculated using the Particle Mesh Ewald (PME) summation scheme. The coulomb interactions and van der Waals were truncated at $1.0 \mathrm{~nm}$. Every 10 steps the non-bonded pair list was updated and every 2 ps conformations were stored. VMD, PyMOL and Gromacs analysis tools were used for analysis of the trajectories $[15,18]$.

\section{RESULTS}

\section{Epitope predictions}

The hirudin sequence obtained from the UniProt $\mathrm{KB}$, was used to predict its promiscuous epitopes using two different prediction programs: ProPred and IEDB. These programs compared the submitted sequences to specific matrices for HLA-DRB1 0101, and predict the binding affinity of the epitopes for the HLAs-DRB1 0101.

Five different epitopes predicted based on previous study were used in this research. In the present study two epitopes from the five epitopes (each epitope consisted of fifteen amino acids) were selected, because they showed a significant likelihood of binding to HLA-DRB1 *0101.

Although the programs are different and have different numerical results, we adopted cut-offs based on the significance of the affinities shown by each epitope. Each of the two programs used for epitope selection has a specific algorithm and was developed in different ways. However, these programs work similarly to predict the strength of the interaction of specific HLAs with proteins and identify epitopes that have greater interactions with this molecule. The epitopes that were obtained for specific HLADRB1*0101 from the two programs were analyzed simultaneously and aligned with the total protein sequence. The aim of this analysis was to combine the best results from the two programs and the final peptide was selected based on residues that were common between both programs. In ProPred, those epitopes that had scores above the default binding threshold were selected. For the IEDB program, those epitopes that had an affinity score below 20 were selected. Of the hirudin analyzed, two sequences containing fifteen amino acids were selected; these corresponded to the epitopes that were most strongly recognized by HLA-DRB1 0101.
To determine their stability, the epitopes were modeled and evaluated. Based on previous study (submitted article) and more study in the present research two regions, I1TYTDCTESGQNLCL15 and L13CLCEGSNVCGKGNK27, were chosen because these appeared to be the most promiscuous among the epitopes examined, and therefore, the most likely to be presented to lymphocytes during a human immune response.

Furthermore, using the IEDB program, and based on in silico mutation of the peptide, mutated epitopes with less antigenicity were determined. Finally [Lys ${ }^{4}$ ] Hir ${ }_{1-15}$ and $\left[\right.$ Gly $^{9}{ }^{9} \mathrm{Hir}_{1-15}$, $\left[\mathrm{Gly}^{21}\right]$ and $\left[\mathrm{Lys}^{21}\right] \mathrm{Hir}_{13-27}$ were selected for further study.

\section{Molecular modeling}

\section{Modeling of epitopes and new mutants of epitopes}

Homology models of selected epitopes and their mutants were constructed with the program MODELLER9v13. Hirudin variant II (PDB code: 4HTC; similarity $89 \%$ with Hirudin variant III) appeared the appropriate template for comparative modeling of epitopes and their mutants of HVIII. The alignment of HVII and epitopes were generated by ClustalW [19]. The 10000 different models generated for each epitopes and mutants by MODELLER were carefully analyzed for energy value and RMSD. Among the models, which were quite similar with energy values, the model possessing the least RMSD value with the backbone atoms of HVII ( $0.02 \AA$ for epitopes and mutants), was selected for further study. The quality of the folding was assessed by VERIFY-3D (http://psvs-1_4dev.nesg.org/), which evaluates the compatibility of a given residue in a certain three-dimensional environment.

PROCHECK [20] analysis indicates that the quality of the Ramachandran plots $(100 \%$ of the residues in the allowed regions) were equivalent to those of the template structure. The superimposition of the modeled epitope 1 (Hir115) and its mutants, epitope 2 (Hir13-27) and its mutants structures is reported in Fig. $1 \mathrm{a}, \mathrm{b}$ and $2 a, b$, respectively.

The selected mutated epitopes were built with the N-terminal mutation, to form the new structures. This strategy was adopted to decrease the immune response, as well as to improve the side effects of these molecules. 

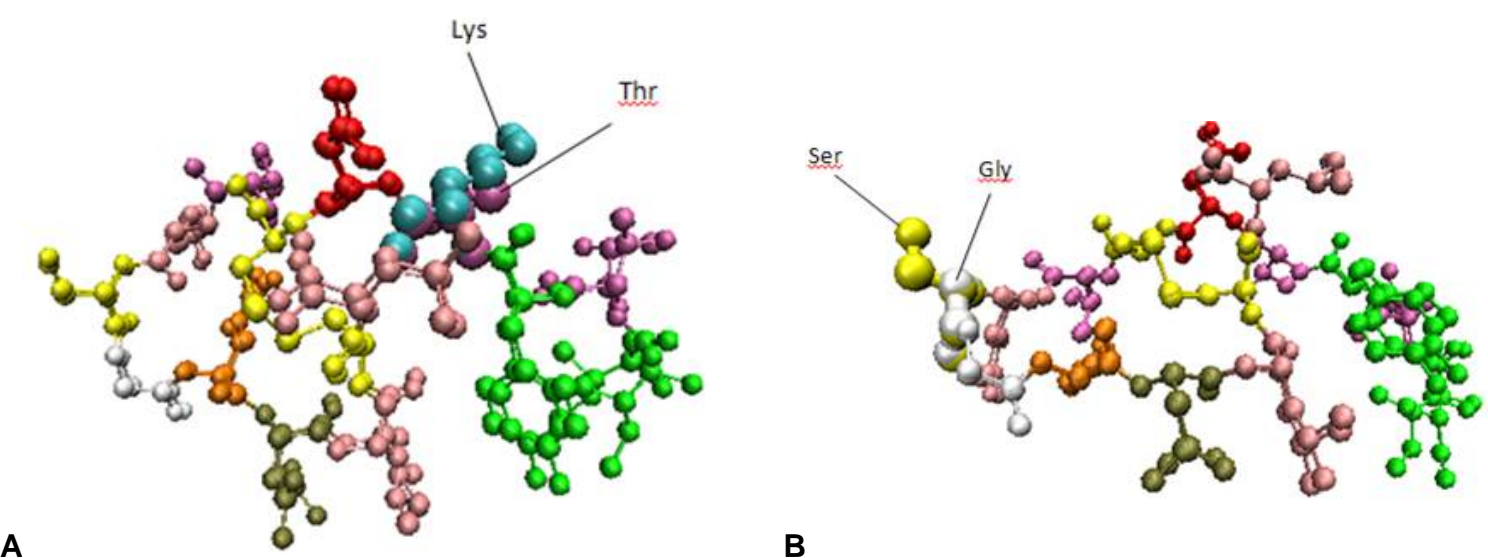

B

Figure 1: Superimposition of hirudin native epitopes and modified epitopes modeled structures (ball and stick shape). (a) native epitope Hir 1-15 ( I TYTDCTESGQNLCL $_{15}$ ) native residue Thr (ball and stick, mauve) and its analog modified peptide [Lys ${ }^{4}$ ] Hir ${ }_{1-15}$ (ball and stick, cyan) (b) native epitope Hir ${ }_{1-15}\left(\mathrm{I}_{1} \mathrm{TYTDCTESGQNLCL}_{15}\right)$ native residue Ser (ball and stick, yellow) and its analog modified peptide [ Gly ${ }^{9}$ ] Hir ${ }_{1-15}$ (white)

A
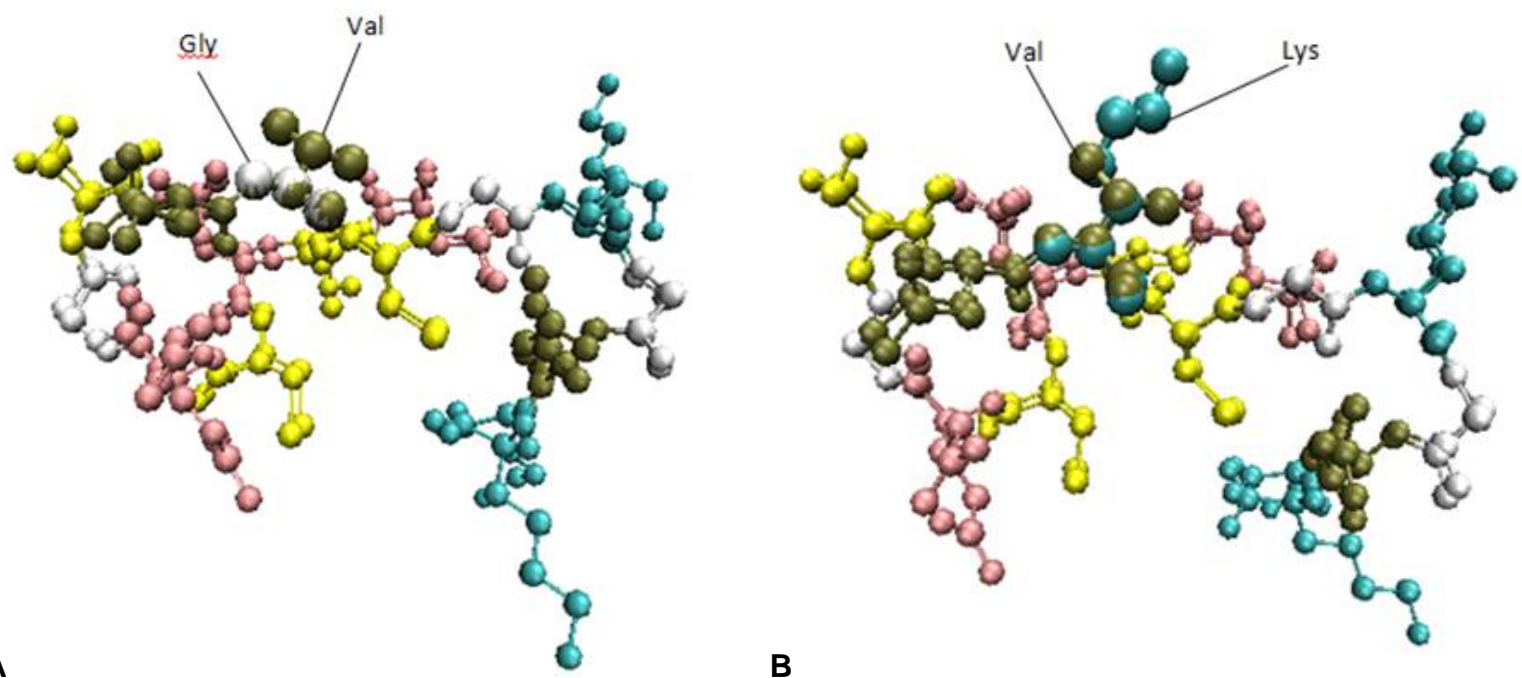

Figure 2: Superimposition of hirudin native epitopes and modified epitopes modeled structures (ball and stick shape). ( $a$ and $b$ ) native epitope $\mathrm{Hir}_{13-27}\left(\mathrm{~L}_{13} \mathrm{CLCEGSNVCGKGNK}_{27}\right)$ native residue Val (ball and stick, tan) and its analog modified peptides [Gly ${ }^{21}$ ] Hir ${ }_{13-27}$ (white) and [Lys ${ }^{21}$ ] Hir ${ }_{13-27}$ (cyan), respectively

The X-ray crystallography data from HLA-DRB1 010 (PDB code: 1PYW) were used to build a model of the complex peptide-HLA1 0101. The major focus of this study was on comparing the dynamic behaviors of native peptides of hirudin and its mutants with lower antigenicity in docked complexes with HLA-DRB1 0101.

The energy minimization method avoids unfavorable atomic interactions and packing forces. Furthermore, the conformational behavior and the potential energy surface were evaluated carefully. The analysis of the torsion angles in the model, which was refined after the MD simulation, showed that this simulation did not cause serious distortions in the structures. The Ramachandran plot indicates that $100 \%$ of their residues are in the allowed regions in all of the epitopes models and their peptide mutants notably, some of the molecules showed conformational changes that formed well-defined secondary structures after the MD calculation, compared with structures generated using comparative modeling. Other epitopes only exhibited small structural changes as a result of the MD simulation.

During the molecular dynamics simulation, the backbone RMSD, which referenced the initial structure, varied more at the beginning of the dynamics simulation and just after reaching the equilibrium state. This result indicates that few structural changes occurred. However, structural variations still occurred throughout the course of 
the simulation. These variations are characteristic of small peptides, which are very flexible and have various minimum energy states, indicating that these peptides can assume different conformations. This structural modification, in turn, is related to the short amino acid sequence, which tends to have a greater degree of freedom. The structural modification also contributes to the large deviations, as observed throughout the MD simulation in Fig 3a and $b$, results in a lack of interaction between the modified epitopes with the amino acid residues in HLA-DRB1 0101 in comparison with native epitopes.
Although there was a variation of $2-3 \AA$ on the average in the RMSD values, there was no ascendant behavior in the graphs, suggesting that these structures reach structural equilibrium despite the flexibility of these epitopes. In addition, the variation in the total energy of the epitopes and their mutants during the 20 ns simulations is presented in Fig. 4a for (Hir) epitope 1-15 and its analogs [Lys ${ }^{4}$ Hir $_{1-15}$ and [Gly ${ }^{9}$ ] Hir ${ }_{1-15}$, in Fig. 4b for (Hir) epitope 13-27, and its analogs [Gly ${ }^{21}$ ] Hir ${ }_{13-27}$ and $\left[\right.$ Lys $^{21}$ ] Hir ${ }_{13-}$ 27 .

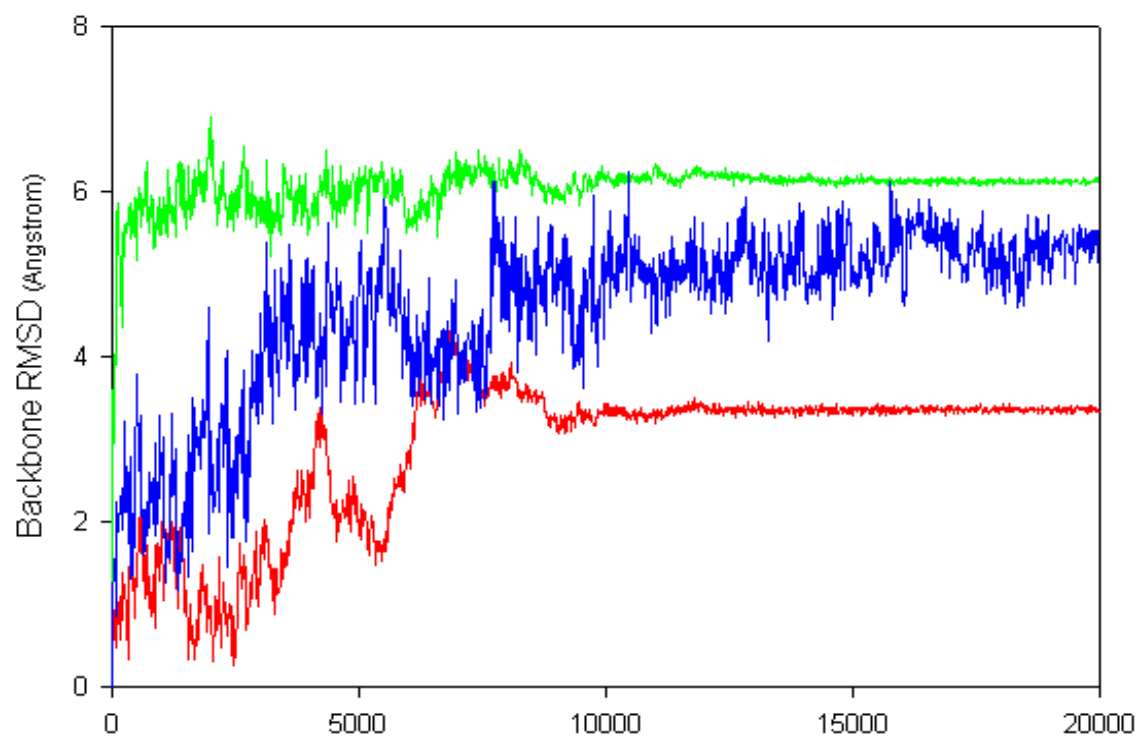

A

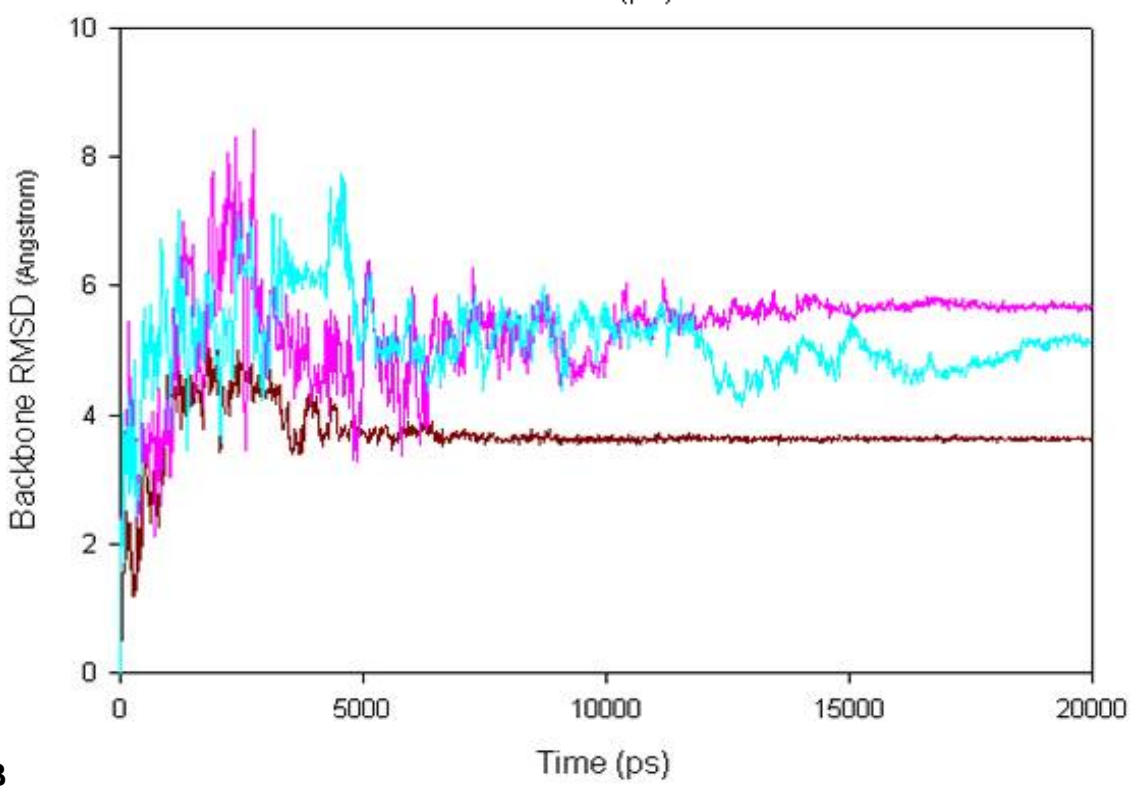

Figure 3: Time evolution of (a) Backbone RMSD (root mean square deviation), this graph, was calculated using a simulation time of $20 \mathrm{~ns}$ for the trajectory of the production. Dynamics, (a) native epitope Hir ${ }^{1-15}$ (red), [Lys ${ }^{4}$ ] Hir ${ }_{1}$ 15 (blue) and $\left[\right.$ Gly $^{9}$ ] Hir ${ }_{1-15}$ (green), (b) native epitope Hir ${ }_{13-27}$ (DK red), [Gly ${ }^{21}$ ] Hir ${ }^{13-27}$ (cyan) and $\left[\text { Lys }^{21}\right]_{\mathrm{Hir}^{13-27}}$ (pink). 
In the studied systems, the mutations promote instability in the epitope and result into high and the more positive energy high of almost 10000 $25000 \mathrm{kcal} / \mathrm{mol}$ in comparison with the native epitopes. Again, this can be due to the lack of interaction of the structure.

\section{DISCUSSION}

Studies based on the identification of potentially immunogenic epitopes of hirudin obtained from medicinal Leech, through rational design, have not only proven to be a promising strategy for safer protein development but have also have aided in the development and improvement of computer programs capable of generating accurate and reliable data $[7,11,21]$. Hirudin has intrinsic factors contributing to antibody formation. Promiscuous epitopes of this protein can stimulate significant proliferation of Th2 lymphocytes, a fact that highlights the utility of this strategy in searching for potentially immunogenic epitopes [22,23].

The advantage of identification of immune dominant epitopes is the ability to design safer protein which would stimulate cellular proliferation to a lesser extent and decrease unwanted immune responses [24].
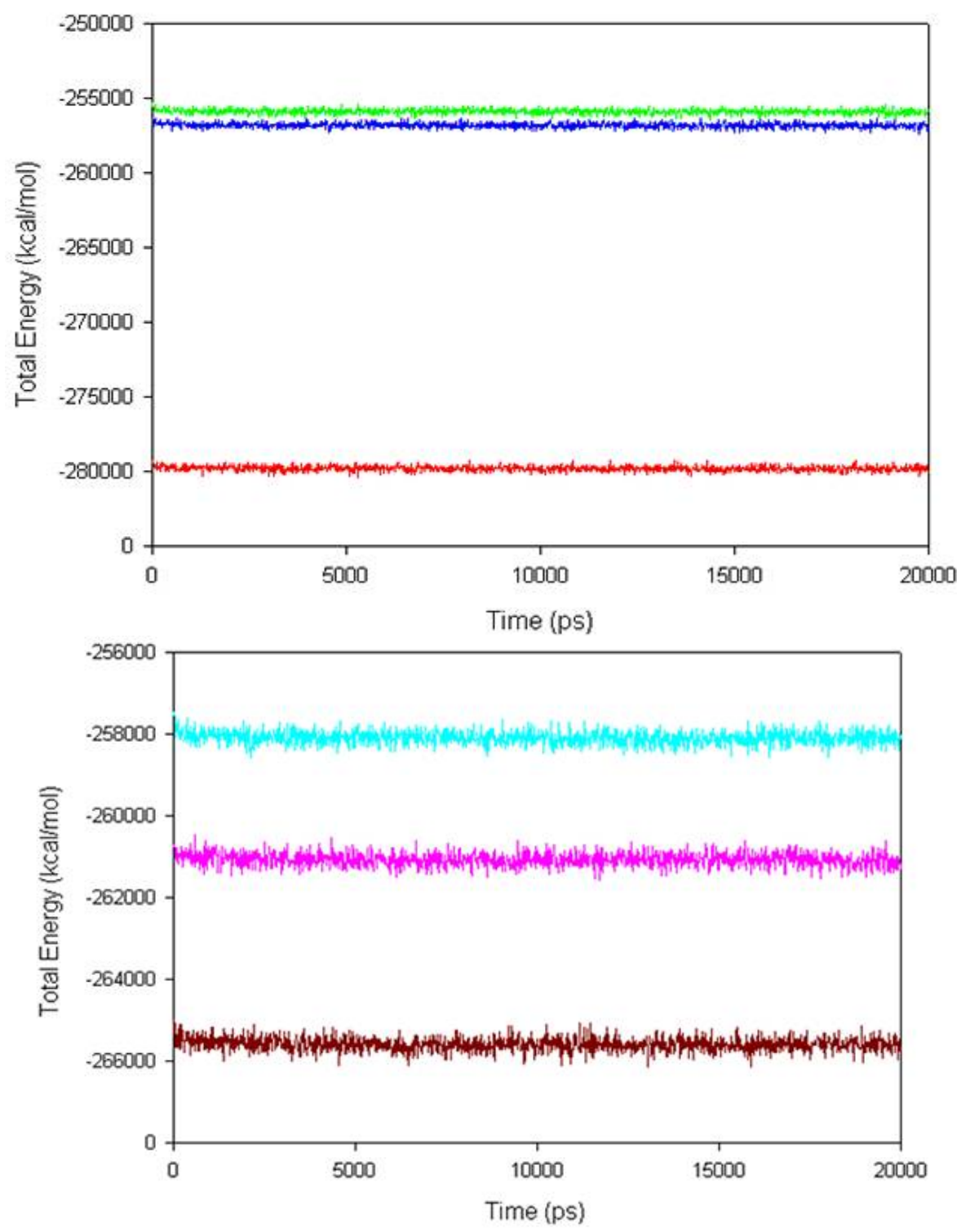

Figure 4: Change in the total energy during the simulation of the production dynamics as a function of time. The simulation was run at $300 \mathrm{~K}$ with a simulation time of $20 \mathrm{~ns}$ for the different epitopes and its modified peptides, (a) native epitope Hir ${ }_{1-15}$ (red), [Lys ${ }^{4}$ ] Hir ${ }_{1-15}$ (blue) and [ Gly ${ }^{9}$ Hir ${ }_{1-15}$ (green), (b) native epitope Hir ${ }_{13-27}$ (DK red), $\left[\mathrm{Gly}^{21}\right]_{\text {Hir }}{ }_{13-27}$ (cyan) and [Lys $\left.{ }^{21}\right]$ Hir ${ }_{13-27}$ (pink) 
In a previous study 3D structure of complete native hirudin and its new mutants (selected based decreased antigenicity) were built, docked with thrombin (as receptor) and molecular dynamic simulation study was done. The results demonstrated that the new mutants were thermodynamically and structural stable (submitted article). But in this study the 3D structures of selected epitopes 1 (Hir ${ }_{1-15}$ ) and 2 (Hir 13-27) and their mutants were built and validated by molecular modeling tools, and the RMSD values of modeled epitopes equaled 0.02 $\AA$, In addition, the stereo chemical quality was analyzed by Ramachandran plot, which determines the distribution of the $\Psi$ and $\Phi$ angle of the main chain. Ramachandran plot of the best epitopes model and their mutants indicated that all residues were located in the favored region.

Finally, the best structures were refined by energy minimization and molecular dynamic simulations. The molecular dynamic studies of native epitopes-HLA-DRB1 0101 and MPLsHLA-DRB1 0101 showed energy and RMSD variation during the simulation. RMSD and energy analysis for the binding site epitopes showed that the binding groove is more stable when HLA-DRB complex with native epitopes in comparison with new mutant epitopes. Overall, these results indicate that new mutants have less propensity for binding to MHC groove and could be considered as safe proteins that have less tendency stimulate the immune system. The use of these approaches has also been shown to be an economically interesting strategy because it is possible to design and verify more mutants.

\section{CONCLUSION}

Molecular dynamic analysis by computational tools is an important technique for antigen identification and characterization for the development protein therapeutics. The immunoinformatic tools used in the present study helps to identify and characterize epitopes to reduce the stimulation of the host immune system. The epitopes and their mutants showed similar structure to those observed in the native proteins; however, they have different thermodynamically stable structures. These studies could improve the prediction level of immunogenicity of native hirudin and its mutants, the induced cytokine profile and the antibodies that are generated.

To the best of our knowledge, the method presented here is novel in predicting hirudin peptide binding to HLA-DRB1 0101 for the design of new mutants that decrease the antigenicity of hirudin.

\section{ACKNOWLEDGEMENT}

The authors wish to thank Ms. Joulia AlizadehRahrovi for her help in the simulation experiments.

\section{REFERENCES}

1. Liu $Y$, Wan $B$, Yan $T-H$, Chang $X-D$, Liao J-M, Wu W-T, Hu R. Effect of Recombinant Hirudin Variant III Emulsive Microparticles on Thrombosis and the Study on Its Oral Absorption Mechanism. Chin J Nat Med 2010; 8: 474-480.

2. Syed S, Schuyler PD, Kulczycky M, Sheffield WP. Potent antithrombin activity and delayed clearance from the circulation characterize recombinant hirudin genetically fused to albumin. Blood 1997; 89: 32433252.

3. Tan S, Wu W, Liu J, Kong $Y, P u Y$, Yuan R. Efficient expression and secretion of recombinant hirudin III in E. coli using the l-asparaginase II signal sequence. Protein Expression Purif 2002; 25: 430-436.

4. Huhle $G$, Hoffmann $U$, Song $X$, Wang $L C$, Heene $D L$, Harenberg J. Immunologic response to recombinant hirudin in HIT type II patients during long-term treatment. Br J Haematol 1999; 106: 195-201.

5. Doolan DL, Southwood S, Chesnut R, Appella E, Gomez E, Richards A, Higashimoto YI, Maewal A, Sidney J, Gramzinski RA. HLA-DR-promiscuous T cell epitopes from Plasmodium falciparum pre-erythrocytic-stage antigens restricted by multiple HLA class II alleles. J Immunol 2000; 165: 1123-1137.

6. Gupta SK, Srivastava M, Akhoon BA, Smita S, Schmitz $U$, Wolkenhauer O, Vera J, Gupta SK. Identification of immunogenic consensus $T$-cell epitopes in globally distributed influenza-A H1N1 neuraminidase. Infect Genet Evol 2011; 11: 308-319.

7. Kumar A, Cocco E, Atzori L, Marrosu MG, Pieroni E. Structural and dynamical insights on HLA-DR2 complexes that confer susceptibility to multiple sclerosis in Sardinia: a molecular dynamics simulation study. PloS one 2013; 8: e59711.

8. Zhang $H$, Wang $P$, Papangelopoulos $N, X u Y$, Sette $A$, Bourne PE, Lund O, Ponomarenko J, Nielsen M, Peters $B$. Limitations of $A b$ initio predictions of peptide binding to MHC class II molecules. PLoS One 2010; 5: e9272.

9. Ghaderi $A$, Talei $A$, Gharesi-Fard $B$, Farjadian $S$, Amirzargar A, Vasei M. HLA-DRB 1 alleles and the susceptibility of Iranian patients with breast cancer. Pathol Oncol Res 2001; 7: 39-41.

10. Safa M, Forouzandeh M, Pourfathollah A, Gill $P$, Rasaee MJ, Yari F, Shahbazi A, Soltanpour MS, Mayor $N$. High Resolution Allotyping of Four Alleles of HLA$D R B 1 * 01$ Group in Iranians Using Reverse-SSOPH

Trop J Pharm Res, December 2015; 14(12): 2177 
Assay in Comparison with DNA Sequencing and PCR-SSP. J Biol Sci 2008; 8.

11. De Oliveira Lopes $D$, de Oliveira FM, do Vale Coelho IE, de Oliveira Santana KT, Mendonça FC, Taranto AG, dos Santos LL, Miyoshi A, de Carvalho Azevedo VA, Comar M. Identification of a vaccine against schistosomiasis using bioinformatics and molecular modeling tools. Infect Genet Evol 2013; 20: 83-95.

12. Asgari S, Mirzahoseini $H$, Karimipour M, Rahimi $H$, Ebrahim-Habibi A. Rational design of stable and functional hirudin III mutants with lower antigenicity. Biologicals in press. Available from: http://dx.doi.org/10.1016/j.biologicals.2015.07.008.

13. Sali A, Blundell TL. Comparative protein modelling by satisfaction of spatial restraints. J Mol Biol 1993; 234 . 779-815.

14. Rydel TJ, Tulinsky A, Bode W, Huber R. Refined structure of the hirudin-thrombin complex. $J$ Mol Biol 1991; 221: 583-601.

15. Van Der Spoel D, Lindahl E, Hess B, Groenhof G, Mark $A E$, Berendsen HJ. GROMACS: fast, flexible, and free. J Comput Chem 2005; 26: 1701-1718.

16. Zavala-Ruiz Z, Sundberg E, Stone J, DeOliveira D, Chan I, Svendsen J, Mariuzza R, Stern L. Human class II $M H C$ protein HLA-DR1 bound to a designed peptide related to influenza virus hemagglutinin, FVKQNA (MAA) $A L$, in complex with staphylococcal enterotoxin C3 variant 3B2 (SEC3-3B2). J Biol Chem 2003; 278: 44904-44912.

17. De Vries SJ, van Dijk M, Bonvin AM. The HADDOCK web server for data-driven biomolecular docking. Nat protocols 2010; 5: 883-897.
18. Humphrey W, Dalke A, Schulten K. VMD: visual molecular dynamics. J Mol Graphics 1996; 14: 33-38.

19. Larkin M, Blackshields G, Brown N, Chenna R, McGettigan PA, McWilliam $H$, Valentin $F$, Wallace IM, Wilm A, Lopez R. Clustal $W$ and Clustal $X$ version 2.0. Bioinformatics 2007; 23: 2947-2948.

20. Laskowski RA, MacArthur MW, Moss DS, Thornton JM. PROCHECK: a program to check the stereochemical quality of protein structures. J Appl Crystallogr 1993; 26: 283-291.

21. Mantzourani ED, Platts JA, Brancale A, Mavromoustakos TM, Tselios TV. Molecular dynamics at the receptor level of immunodominant myelin basic protein epitope 87-99 implicated in multiple sclerosis and its antagonists altered peptide ligands: triggering of immune response. J Mol Graphics Modell 2007; 26: 471-481.

22. Borras-Cuesta F, Golvano J-J, Garc a-Granero $M$, Sarobe P, Riezu-Boj J-I, Huarte E, Lasarte J-J. Specific and general HLA-DR binding motifs: comparison of algorithms. Hum immuno 2000; 61: 266-278.

23. Tangri S, LiCalsi C, Sidney J, Sette A. Rationally engineered proteins or antibodies with absent or reduced immunogenicity. Curr Med Chem 2002; 9: 2191-2199.

24. Tangri S, MothÃc BR, Eisenbraun J, Sidney J, Southwood $S$, Briggs $K$, Zinckgraf J, Bilsel $P$, Newman $M$, Chesnut R. Rationally engineered therapeutic proteins with reduced immunogenicity. $J$ Immunol 2005; 174: 3187-3196. 